\title{
Comparación analítica de arreglos de antenas Yagi por los métodos de momentos y multiplicación de patrones
}

\author{
R. Neri-Vela, L.A. Valiente-Montaño y V. Hernández-Solís \\ Departamento de Telecomunicaciones \\ Facultad de Ingeniería, UNAM \\ E-mails: aldeca@telecom.fi-b.unam.mx, $\underline{\text { a valiente@correo.unam.mx } y}$ vladimirher@correo.unam.mx
}

(recibido: agosto de 2002; aceptado: marzo de 2003)

\section{Resumen}

\begin{abstract}
Las propiedades de operación de arreglos horizontales y verticales formados con tres antenas Yagi son analizadas por el método de momentos, considerando la impedancia mutua entre ellas, para Yagis con número creciente deelementos (3, 6 y 9), optimizando la impedancia total de entrada de cada arreglo a una frecuencia central de $500 \mathrm{MHz}$. Adicionalmente, el patrón de radiación obtenido para cada caso es comparado con la aproximación dada por d método de multiplicación de patrones, variando la separación entre las Yagis para conocer hasta qué distancia influye significativamente la impedancia mutua entre los elementos del arreglo. Además de ser útil comercialmente, esta información sirve como material didáctico para la asignatura de Antenas enlas carreras de Ingeniería.
\end{abstract}

Descriptores: Método de momentos, método de multiplicación de patrones, arreglo de antenas, antenas Yagi, Troica.

\begin{abstract}
The operational properties of horizontal and vertical arrays formed by 3 Yagi antennas are analized by the Method of Moments (MoM), taking into account the mutual impedances between them. Yagis with an increasing number of elements (3, 6 and 9 ) are used, optimizing the input impedance of each arra $y$ at a fixed center frequency of $500 \mathrm{MHz}$. The radiation pattern obtained in each case is compared to the approximation given by the Pattern Multiplication Method for different separations between the Yagi antennas of each arra y, to determine the influence of distante in the calculation of the radiation characteristics. Besides being commercially useful, this information is also of teaching value on the subject of antennas in telecommunications engineering.
\end{abstract}

Keywords: Method of moments, pattern multiplication method, antenna arrays, Yagi antennas.

\section{Introducción}

Las antenas Yagi, como la mostrada en la figura 1, pueden ser utilizadas de forma sencilla en aplicaciones tales como recepción de señales de TV en las bandas de VHF y UHF, repetidores en las bandas de telefonía celular y PCS, en 800 y 1900 $\mathrm{MHz}$ respectivamente, comunicaciones rurales, etc.
La ganancia que proporcionan estas antenas depende del número de elementos parásitos que las componen y se encuentra típicamente entre 6 y $15 \mathrm{dBi}$, según los requerimientos de su aplicación; (Balanis, 1997; Decibel Products, 2000).

Sin embargo, existen casos en la práctica donde es necesario incrementar la directividad de la antena, y para ello, se recurre a formar 
Comparación analítica de arreglos de antenas Yagi por los métodos de momentos...

arreglos integrados con dos o más antenas elementales idénticas, que pueden ser del tipo Yagi, dipolos sencillos o doblados, platos parabólicos, etc. Si la separación entre dichas antenas elementales y las alimentaciones (magnitud y fase) de cada una de ellas son elegidas adecuadamente, entonces el arreglo resultante ofrece las ventajas buscadas de mayor directividad. En el caso de las antenas Yagi es muy común utilizar arreglos de dos, tres o cuatro antenas elementales para incrementar la directividad en comunicaciones de control o punto a punto (Decibel Products, 2000). Uno de estos arreglos, también conocido como troica (Rossier, 1986) consiste de tres antenas Yagi que pueden estar colocadas formando una cortina horizontal (Figura 2) o una cortina vertical (Figura 3). No obstante, la información relativa a sus características de operación es escasa en los folletos de fabricantes y prácticamente nula en los libros de texto. Es evidente que si sus separaciones y alimentaciones son seleccionadas correctamente, la ganancia de cada arreglo debe ser mayor que la de una sola antena elemental; pero para realizar una alimentación correcta, es necesario conocer la impedancia de entrada de cada una, y ésta depende del acoplamiento mutuo de impedancias entre todos los conductores del arreglo. Es de esperarse que esta impedancia mutua se reduzca de antena a antena conforme aumente la separación $D$ mos- trada en las figuras 2 y 3. La única forma de saber el efecto de dicha separación sobre el acoplamiento entre las Yagis y, por ende, sobre las impedancias de entrada y los patrones de intensidad de radiación relativa de cada antena elemental es usando el método de momentos (Harrington, 1968; Neri, 1999) o realizando exhaustivas mediciones. Estas últimas no son fáciles de llevar a cabo, pues aún si se contase con una cámara anecoica, se requeriría de una estructura de montaje a la vez rígida y con la flexibilidad suficiente para incrementar progresivamente la separación $D$ entre las antenas.

Por lo anterior, en el presente trabajo se han analizado varias cortinas horizontales y verticales por el método de momentos, variando progresivamente la separación $D$ y usando como antenas elementales para cada arreglo Yagis con 3, 6 y 9 elementos (1 reflector, 1 dipolo doblado y 1,4 y 7 directores, respectivamente). La separación D influye en el patrón de radiación resultante; no puede ser demasiado grande (del orden de varias longitudes de onda) y también hay que evitar que las antenas hagan contacto entre sí, de tal manera que un valor típico recomendado con base en mediciones prácticas es de aproximadamente $0.75 \lambda$, (Rossier, 1986), por lo cual, se modelaron arreglos con valores de $D$ entre $0.5 \lambda$, y $1.0 \lambda$,

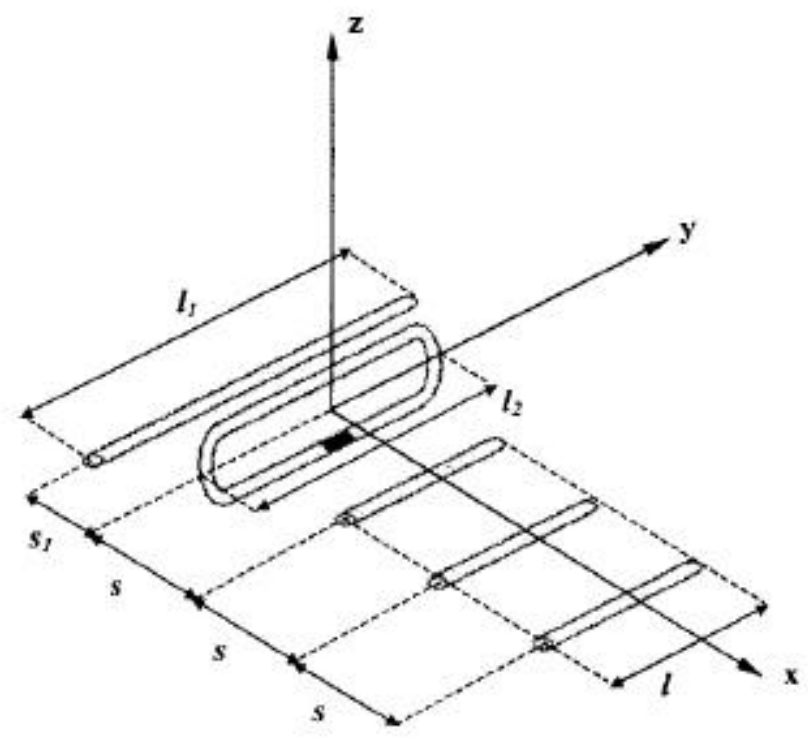

Figura I. Antena Yagi alimentada con un dipolo doblado transversal. La zona negra indica el sitio de conexión al cable de alimentación 
R. Neri-Vela, L.A. Valiente-Montaño y V. Hernández-Solís

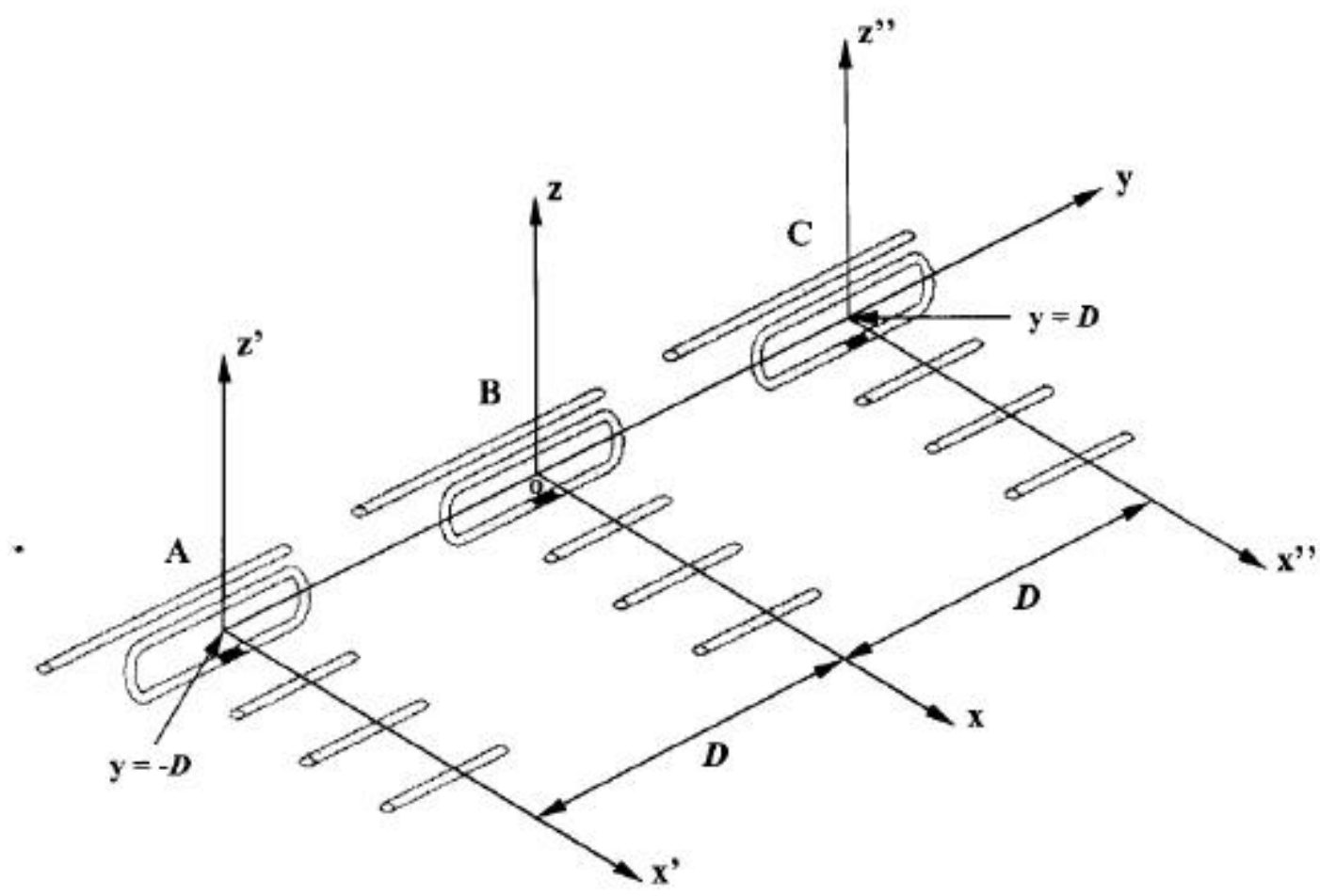

Figura 2. Cortina fiorizontal de tres antenas Yagi (A, B y C) alimentadas con un dipolo doblado contenido en el plano yz

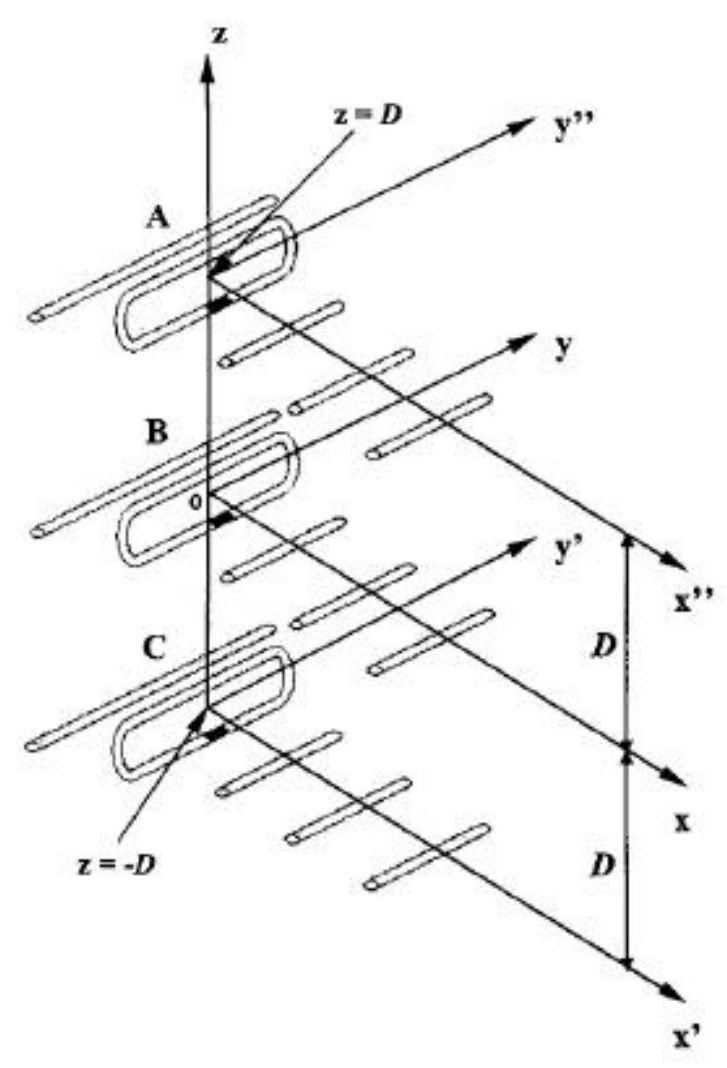

Figura 3. Cortina vertical de tres antenas Yagi (A, B y C) alimentadas con un dipolo doblado contenido en el plano yz 
Comparación analítica de arreglos de antenas Yagi por los métodos de momentos...

Los arreglos de antenas Yagi, además de brindar mayor ganancia que una antena sola, ya que el ancho de su haz principal es más angosto, tienen además aplicaciones muy importantes en comunicaciones ionosféricas y en la recepción de TV en áreas con alta interferencia electromagnética. Esto se debe a que, al montar las Yagis sobre un pedestal muy alto, el efecto del plano de tierra sobre el patrón final del arreglo es reducir el ángulo de inclinación del lóbulo principal, con respecto al plano del terreno, varios grados menos que el ángulo de inclinación que tendría el lóbulo principal de una Yagi sola, cuando también se toma en cuenta el efecto del plano de tierra.

Por lo anterior, muchos radioaficionados en todo el mundo utilizan arreglos de Yagis con el fin de comunicarse a miles de kilómetros de distancia, usando como puntos de reflexión las capas ionosféricas (http://www.uksmg.org/stack.htm). Mientras menor sea el ángulo de ataque del lóbulo principal del arreglo sobre dichas capas, se obtiene mayor alcance. En cuanto a su aplicación en la recepción de TV, los arreglos de Yagis, además de mejorar la ganancia, reducen el ancho del haz principal, y con ello, se reduce la probabilidad de recibir interferencias (con la consiguiente aparición de "fantasmas"). Estas interferencias pueden provenir de reflexiones con vehículos terrestres, aviones, edificios $\mathrm{y}$ otros obstáculos (http://pages.cthome,net/fmdx/stackant.html).

Como se mencionó anteriormente, en el presente trabajo se estudian las propiedades de varias cortinas o arreglos de antenas Yagis. Es importante aclarar que en este estudio se consideró que los arreglos están en el espacio libre y, por lo tanto, no se tomó en cuenta el efecto del plano de tierra. Sin embargo, con los resultados y la metodología que se aportan, dicho efecto puede calcularse con el método de imágenes. No se incluye en este trabajo porque la extensión del artículo sería excesiva, dado que habría que usar otros parámetros como tipo del terreno, altura del arreglo sobre el piso, tipo de polarización, etc., y puede ser objeto de otro estudio posterior.

Los patrones de radiación obtenidos por el método de momentos serán comparados con los patrones que serían predichos por el método gráfico de multiplicación de patrones. Este último método no toma en cuenta los acoplamientos de impedancia entre los conductores de las Yagis de cada arreglo y, por tanto, sólo da una visión aproximada del patrón de radiación. Sin embargo, como se demostrará en este trabajo, dicha aproximación tiende a empeorar cuando las antenas del arreglo están muy próximas entre ellas. De ahí que en este artículo se aporta información nueva en lo que se refiere a la precisión del método de multiplicación de patrones en comparación con el método de momentos, en función de la separación eléctrica entre las antenas elementales.

\section{Desarrollo por el método de momentos}

No existe una regla única ni un desarrollo matemático que permita encontrar directamente las características de radiación de una antena Yagi, y menos de un arreglo, ya que se pueden obtener buenos resultados de operación con una gama de diferentes longitudes y separaciones entre los elementos parásitos (Balanis, 1997). Sin embargo, existen referencias experimentales de parámetros típicos sobre la longitud de dichos parásitos y la separación entre ellos (Balanis, 1997); (Kraus et al., 2002), que dan resultados óptimos cuando dichas antenas trabajan en forma aislada o individual. Si se toman en cuenta estos parámetros típicos como punto de partida, es posible ajustarlos mediante un programa de computadora (Hernández et al.. 2002) diseñado especialmente para tal propósito, con el fin de optimizar la impedancia de entrada. Dicha impedancia se considera óptima cuando su parte real es cercana a $75 \Omega$ y su parte imaginaria despreciable, lo cual garantiza un acoplamiento ideal entre la antena aislada y un cable de alimentación con impedancia característica comercial de $75 \Omega$. Este programa de computadora fue elaborado por los mismos autores de este artículo para el análisis de antenas Yagi que trabajan de forma individual y se utilizó para calcular las longitudes óptimas $1_{1}$ y $1_{2}$ que se muestran en las tablas 1 y 2 para cada antena de cada arreglo. Como facilidad para el lector interesado, el sitio (Hernández et al., 2002) donde dicho programa puede ser descargado, también contiene todas las fórmulas y el procedimiento matemático del método de momentos aplicado a antenas Yagi.

Además de los parámetros de las antenas analizadas, en las tablas 1 y 2 también se muestra la impedancia de entrada $(R+j X)$ calculada para cada Yagi, así como la impedancia de entrada total 
$\left(\mathrm{R}_{\mathrm{i}}+{ }_{\mathrm{j}} \mathrm{X}_{\mathrm{i}}\right)$ del circuito de alimentación de cada troica y la ganancia y FBR de cada arreglo. Por lo que se refiere al circuito de alimentación, se ha demostrado experimentalmente que la forma más efectiva de conectar las tres antenas elementales con el cable de alimentación es como se muestra en la figura 4 (Rossier, 1986). En ella se observa que la impedancia de entrada de las Yagis en los extremos (A y C) de las figuras 2 y 3 quedan en serie y su resultante, a la vez, queda en paralelo con la impedancia de entrada de la antena elemental
central_(B). De ahí que los voltajes de alimentación utilizados en el método de momentos (Neri, 1999) se eligieron como $\mathrm{V}_{\mathrm{B}}=1<0^{\circ}$ y $\mathrm{V}_{\mathrm{A}}=\mathrm{V}_{\mathrm{C}}=\mathrm{V}_{\mathrm{B}} / 2=0.5<0^{\circ}$

De acuerdo con la simetría con respecto al plano $x z$ (Figura 2), las impedancias de entrada de las antenas Yagi en los extremos de una cortina horizontal son iguales y sólo es necesario reportar una de ellas en la tabla 1; en cambio, en la tabla 2, sí se muestran los valores de impedancia de entrada de las tres antenas del arreglo, puesto que no existe la condición de simetría con respecto al plano $x y$ (Figura3).
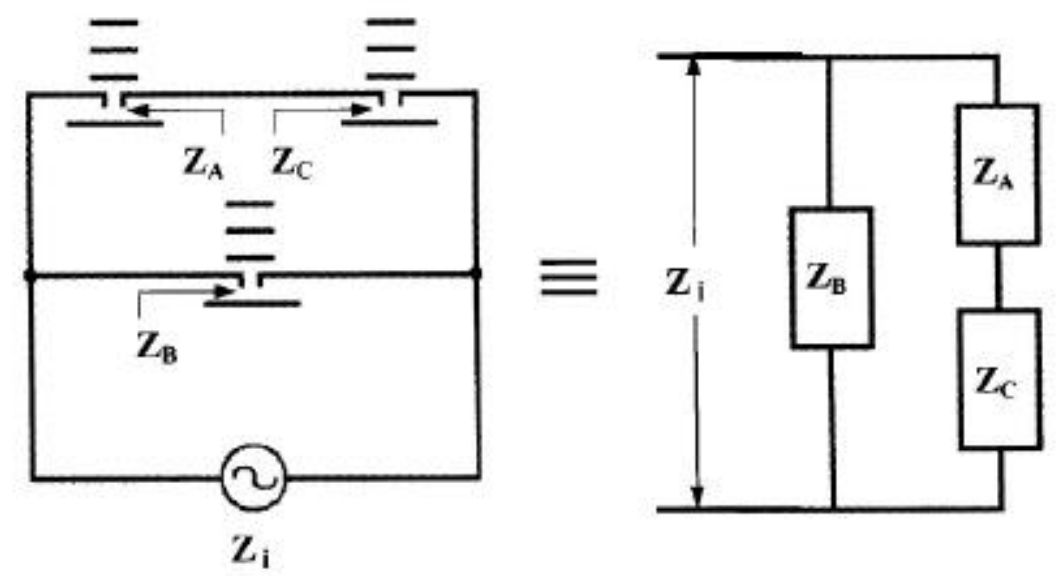

Figura 4. Circuito equivalente para el cálculo de la impedancia de entrada $\left(Z_{i}\right)$ de cada arreglo (horizontal o vertical). siguiendo la recomendación (Rossier, 1986) para obtener resultados óptimos en la práctica. Por simplicidad, cada antena Yagi del arreglo está representada sólo con tres directores

Tabla 1. Cortina horizontal. Los valores presentados fueron obtenidos a una frecuencia de trabajo de $500 \mathrm{Mhz}$ con $1=0.42 ., \mathrm{s}_{1}=0.22$, y s=0.32, para todos los casos. Por la simetría del arreglo, zC=ZA (Figura 4$)$

\begin{tabular}{|c|c|c|c|c|c|c|c|c|c|c|c|}
\hline $\begin{array}{l}\text { Número } \\
\text { elementos } \\
\text { en cada } \\
\text { Yagi }\end{array}$ & $1_{1}[\lambda]$ & $\mathbf{l}_{2}[\lambda]$ & $\mathrm{D}[\lambda]$ & $\mathrm{G}\left[\mathrm{dB}_{\mathrm{i}}\right]$ & $\mathrm{R}_{\mathrm{A}}[\Omega]$ & $\mathbf{X}_{\mathrm{A}}[\Omega]$ & $\mathrm{R}_{\mathrm{B}}[\Omega]$ & $\mathrm{X}_{\mathrm{B}}[\Omega]$ & $\mathrm{R}_{\mathrm{i}}[\Omega]$ & $\mathrm{X}_{\mathrm{i}}[\Omega]$ & $\mathrm{FBR}[\mathrm{B}]$ \\
\hline 3 & 0.49 & 0.439 & 0.50 & 14.0 & 150.7 & 24.5 & 120.0 & -9.3 & 86.8 & -0.9 & 8.6 \\
\hline 6 & 0.47 & 0.447 & 0.50 & 18.8 & 121.8 & 16.7 & 89.5 & -6.7 & 66.0 & -1.2 & 11.0 \\
\hline 3 & 0.49 & 0.415 & 0.60 & 12.4 & 119.7 & 8.7 & 119.9 & -3.2 & 80.1 & 0.5 & 8.6 \\
\hline 6 & 0.47 & 0.417 & 0.60 & 15.0 & 107.6 & 1.8 & 121.3 & -1.1 & 77.6 & 0.0 & 7.9 \\
\hline 9 & 0.49 & 0.415 & 0.60 & 16.5 & 118.6 & -2.3 & 131.9 & 2.5 & 84.8 & 0.4 & 10.5 \\
\hline
\end{tabular}


DOI: http://dx.doi.org/10.22201/fi.25940732e.2005.06n2.006

Tabla 1. Cortina horizontal. Los valores presentados fueron obtenidos a una frecuencia de trabajo de 500 Mhz con $1=0.4 \lambda, \quad \mathrm{s}_{1}=0.2 \lambda$, y $\mathrm{s}=0.3 \lambda$ para todos los casos. Por la simetría del arreglo, $\mathrm{Z}_{\mathrm{C}}=\mathrm{Z}_{\mathrm{A}}$ (Figura 4) (...continuación)

\begin{tabular}{|c|c|c|c|c|c|c|c|c|c|c|c|}
\hline $\begin{array}{l}\text { Número } \\
\text { de } \\
\text { elementos } \\
\text { en cada } \\
\text { Yagi }\end{array}$ & $1_{1}[\lambda]$ & $1_{2}[\lambda]$ & $\mathrm{D}[\lambda]$ & $\mathrm{G}[\mathrm{dBi}]$ & $\mathrm{R}_{\mathrm{A}}\lceil\Omega\rceil$ & $\mathrm{X}_{\mathrm{A}}[\Omega]$ & $\mathrm{R}_{\mathrm{B}}[\Omega]$ & $\mathrm{X}_{\mathrm{B}}[\Omega]$ & $\mathrm{R}_{\mathrm{i}}[\Omega]$ & $\mathrm{X}_{\mathrm{i}}[\Omega]$ & $\mathrm{FBR}[\mathrm{dB}]$ \\
\hline 3 & 0.49 & 0.410 & 0.75 & 12.7 & 111.1 & 1.0 & 110.0 & 1.0 & 73.6 & 1.0 & 10.0 \\
\hline 6 & 0.47 & 0.417 & 0.75 & 14.5 & 104.4 & 2.5 & 110.8 & -1.6 & 72.4 & -0.1 & 8.5 \\
\hline 9 & 0.49 & 0.414 & 0.75 & 15.4 & 120.1 & -1.3 & 138.3 & 1.1 & 87.8 & 0.1 & 11.6 \\
\hline 3 & 0.49 & 0.409 & 0.90 & 12.8 & 110.9 & 0.7 & 110.6 & 0.7 & 73.8 & 0.5 & 10.4 \\
\hline 6 & 0.47 & 0.415 & 0.90 & 13.8 & 94.2 & -0.3 & 91.6 & 2.0 & 61.7 & 0.7 & 8.2 \\
\hline 9 & 0.49 & 0.416 & 0.90 & 15.6 & 115.0 & 16.5 & 120.8 & -2.8 & 79.2 & -0.8 & 12.8 \\
\hline 3 & 0.49 & 0.409 & 1.00 & 12.8 & 111.1 & 0.7 & 111.0 & 0.8 & 74.0 & 0.5 & 10.1 \\
\hline 6 & 0.47 & 0.416 & 1.00 & 14.9 & 98.0 & 0.8 & 97.9 & -0.1 & 65.3 & 0.2 & 9.9 \\
\hline 9 & 0.49 & 0.416 & 1.00 & 15.6 & 111.8 & 2.1 & 113.2 & -1.1 & 75.2 & 1.7 & 13.3 \\
\hline
\end{tabular}

Tabla 2. Cortina vertical. Los valores presentados fueron obtenidos a una frecuencia de trabajo de $500 \mathrm{Mhz}$ con $1=0.4 \mathrm{X}$, $s_{1}=0.2 \mathrm{X}$ y $\mathrm{s}=0.3$ ?, para todos los casos. Aquí $Z_{c}^{\prime} \# Z_{\mathrm{A}}$ (Figura 4)

\begin{tabular}{|c|c|c|c|c|c|c|c|c|c|c|c|c|c|}
\hline $\begin{array}{c}\text { Número } \\
\text { de } \\
\text { elementos } \\
\text { en cada } \\
\text { Yagi }\end{array}$ & $1_{1}[\lambda]$ & $1_{2}[\lambda]$ & $\mathrm{D}[\lambda]$ & $\mathrm{G}[\mathrm{dBi}]$ & $\mathrm{R}_{\mathrm{A}}[\Omega]$ & $\mathrm{X}_{\mathrm{A}}[\Omega]$ & $\mathrm{R}_{\mathrm{B}}[\Omega]$ & $\mathrm{X}_{\mathrm{B}}[\Omega]$ & $\mathrm{R}_{\mathrm{c}}[\Omega]$ & $\mathrm{X}_{\mathrm{c}}[\Omega]$ & $\mathrm{R}_{\mathrm{i}}[\Omega]$ & $\mathrm{X}_{\mathrm{i}}[\Omega]$ & $\mathrm{FBR}[\mathrm{dB}]$ \\
\hline 3 & 0.49 & 0.410 & 0.50 & 13.0 & 113.5 & 24.2 & 110.0 & -2.8 & 110.9 & 1.1 & 73.9 & -0.9 & 12.5 \\
\hline 6 & 0.47 & 0.414 & 0.50 & 14.1 & 112.5 & -1.6 & 135.0 & 4.0 & 109.0 & -3.0 & 83.9 & 0.9 & 8.1 \\
\hline 9 & 0.49 & 0.404 & 0.50 & 16.4 & 128.7 & -18.7 & 140.1 & 13.3 & 123.7 & -20.5 & 91.4 & 0.6 & 15.2 \\
\hline 3 & 0.49 & 0.409 & 0.60 & 13.6 & 112.2 & -0.5 & 110.2 & 1.8 & 110.7 & 1.0 & 73.7 & 0.8 & 16.4 \\
\hline 6 & 0.47 & 0.419 & 0.60 & 14.6 & 110.0 & 3.6 & 119.6 & -4.3 & 108.2 & 5.3 & 77.4 & -0.7 & 9.7 \\
\hline 9 & 0.49 & 0.415 & 0.60 & 15.9 & 139.8 & -2.4 & 179.0 & 0.1 & 137.2 & 1.0 & 108.7 & -0.2 & 15.3 \\
\hline 3 & 0.49 & 0.410 & 0.75 & 12.7 & 112.2 & 1.5 & 113.4 & -1.3 & 113.0 & 2.8 & 75.4 & -0.1 & 8.78 \\
\hline 6 & 0.47 & 0.418 & 0.75 & 14.8 & 98.6 & 3.3 & 96.9 & -3.3 & 99.3 & 4.6 & 65.1 & -0.6 & 10.5 \\
\hline 9 & 0.49 & 0.421 & 0.75 & 15.1 & 128.3 & 10.5 & 128.3 & -4.0 & 129.4 & 12.7 & 85.9 & 0.8 & 10.1 \\
\hline 3 & 0.49 & 0.408 & 0.90 & 12.4 & 107.7 & -1.2 & 106.2 & --0.4 & 108.5 & -2.5 & 71.2 & -0.6 & 8.2 \\
\hline 6 & 0.47 & 0.415 & 0.90 & 13.8 & 94.2 & -0.3 & 91.6 & 2.0 & 94.8 & -1.2 & 61.7 & 0.7 & 8.2 \\
\hline 9 & 0.49 & 0.414 & 0.90 & 14.6 & 112.9 & 1.6 & 112.0 & -0.9 & 113.5 & -0.1 & 74.9 & -0.2 & 10.6 \\
\hline 3 & 0.49 & 0.408 & 1.00 & 12.8 & 110.7 & -0.4 & 110.5 & 0.4 & 109.9 & -1.2 & 73.6 & 0.0 & 9.8 \\
\hline 6 & 0.47 & 0.414 & 1.00 & 14.2 & 96.9 & -1.3 & 98.1 & 0.1 & 96.3 & -2.0 & 65.1 & -0.3 & 9.3 \\
\hline 9 & 0.49 & 0.413 & 1.00 & 14.9 & 116.3 & -0.5 & 113.4 & -0.8 & 115.2 & -1.1 & 76.1 & -0.5 & 12.8 \\
\hline
\end{tabular}


Sin embargo, la diferencia entre estas impedancias de las antenas Yagi en los extremos de la cortina vertical son muy pequeñas (del orden de algunos Ohms), de modo que, como se ve más adelante en la sección del método de multiplicación de patrones, es posible considerar que la magnitud de radiación es la misma en las antenas Yagi que se encuentran en los extremos de una troica.

Aun cuando la ganancia obtenida para una separación $\mathrm{D}=0.75 \lambda$ no es siempre la mejor, es menor a la de otras separaciones solamente por algunas décimas de decibel, y de las figuras 7 y 8 se observa que para distancias menores se tiene un ancho de haz principal mayor, por lo tanto, una menor directividad; en cambio, para separaciones mayores, se incrementa el número de lóbulos laterales, lo cual generalmente no es deseable. Por estas razones, y por cuestiones de espacio, en la figura 5 se muestra la distribución de ganancia en función de la frecuencia para cada uno de los arreglos modelados, pero solamente para una separación $\mathrm{D}=0.75 \lambda$.

Se demuestra que la ganancia de una troica, ya sea horizontal o vertical, es siempre mayor a la de una antena Yagi elemental con el mismo número de directores, independientemente de la frecuencia a la que se trabaje dentro del intervalo estudiado (480 $\mathrm{MHz}$ a $520 \mathrm{MHz}$ ). En cuanto a su ganancia, no es fácil decidir si una troica se comporta mejor con una cortina horizontal que una vertical, puesto que ambas tienen ganancias similares y los pares de curvas son distintos según el número de directores que tenga cada Yagi, aunque la cortina horizontal tiene una ganancia mayor en las frecuencias superiores a la frecuencia central. Todos los arreglos aquí mostrados tienen una ganancia relativamente estable con respecto a la frecuencia, puesto que la máxima diferencia entre sus valores máximo y mínimo es de 2 $\mathrm{dB}$, por lo que se puede afirmar que el ancho de banda de $3 \mathrm{~dB}$ con respecto a la ganancia máxima es mayor que la banda mostrada en la figura 5. Sin embargo, también se puede definir el ancho de banda como una función del VSWR (Voltage Standing Wave Ratio) o Relación de Onda Estacionaria, el cual está dado por:

$$
V S W R=\frac{1+|\rho|}{1-p \mid}
$$

donde el coeficiente de reflexión p se define como:

$$
\rho=\frac{Z_{i}-75}{Z_{i}+75}
$$

considerando una impedancia característica de $75 \Omega$ para el cable de alimentación. Un acopla miento de impedancias se considera aceptable para valores de VSWR menores que 1.5 (Collin, 1985). En la figura 6 se muestra el VSWR de los arreglos con respecto a la frecuencia; se observa que el VSWR más alto se obtiene para las antenas Yagi. Esto sucede porque no fueron optimizadas para igualar su impedancia de entrada a $75 \Omega$, sino que simplemente se utilizaron las dimensiones de las troicas pero en antenas aisladas. En lo que respecta a las troicas, una cortina horizontal de 3 elementos tiene aproximadamente el mismo ancho de banda que su análogo vertical; mientras que para unnúmero mayor de elementos $(6$ o 9) el ancho de banda del arreglo horizontal es mayor que el del vertical por 6-7 MHz. Regresando a las figuras 7 y 8 en ellas también se muestra el patrón de radiación obtenido por el método de multiplicación de patrones, superpuesto al patrón predicho por el método de momentos. 
G [dBi]

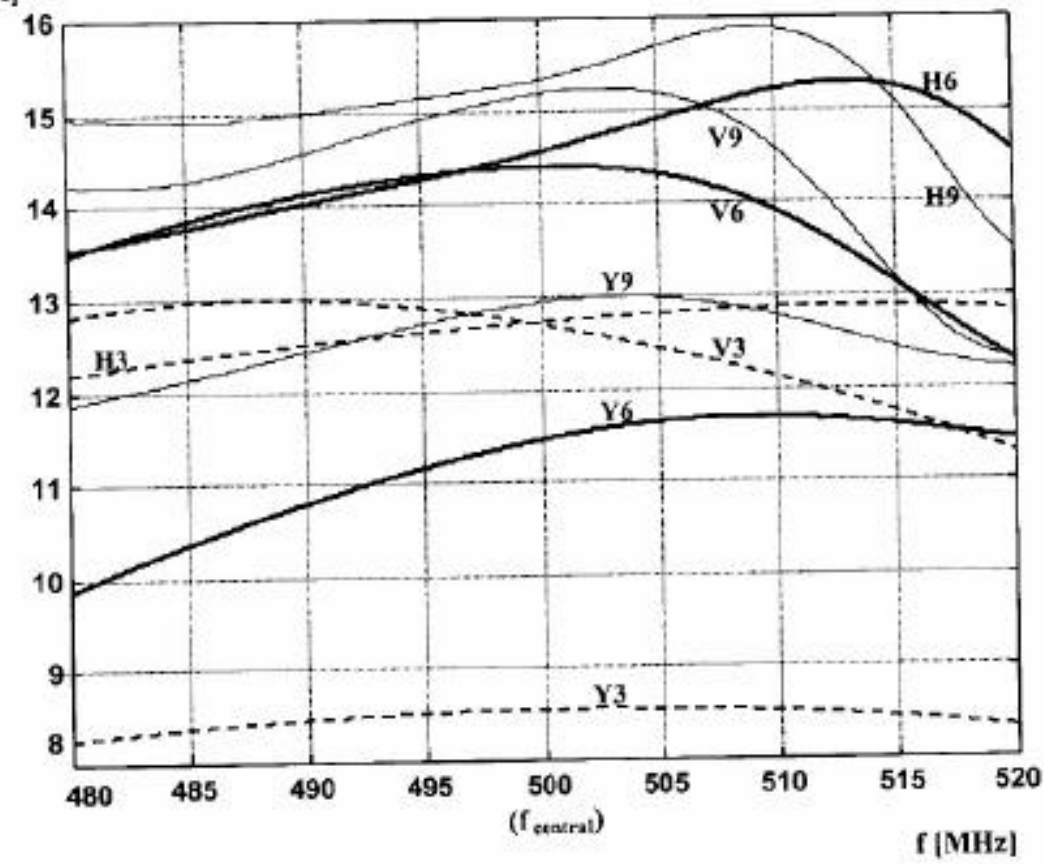

Figura 5. Ganancia de: Yagi única con $\mathrm{N}$ elementos (YN), cortinas horizontales con tres Yagis de $\mathrm{N}$ elementos (HN) y cortinas verticales con tres Yagis de $\mathrm{N}$ elementos (VN)

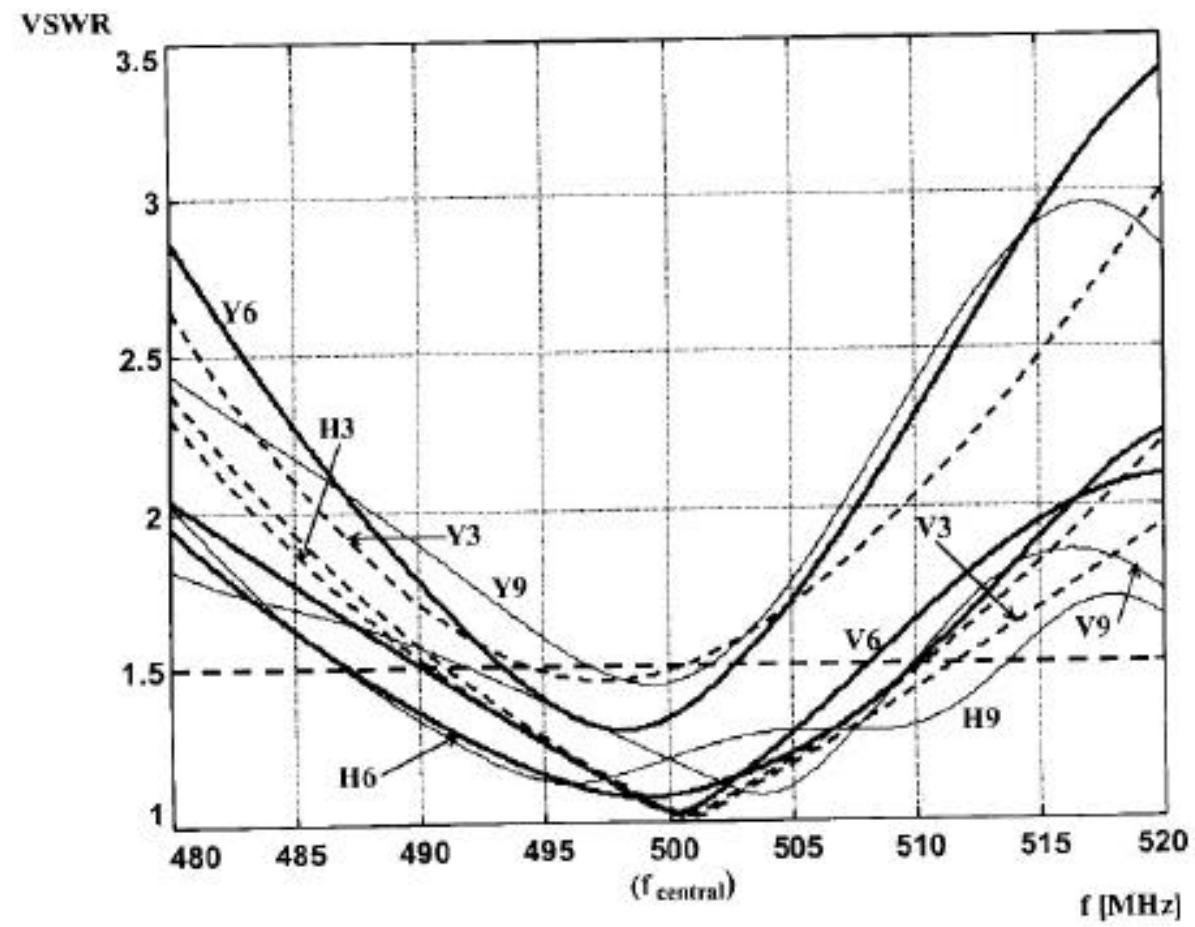

Figura 6.VSWR de: Yagi única con N elementos (YN), cortinas horizontales con tres Yagis de $\mathrm{N}$ elementos (HN) y cortinas verticales con tres Yagis de $\mathrm{N}$ elementos (VN) 
R. Neri-Vela, L.A. Valiente-Montaño y V. Hernández-Solís

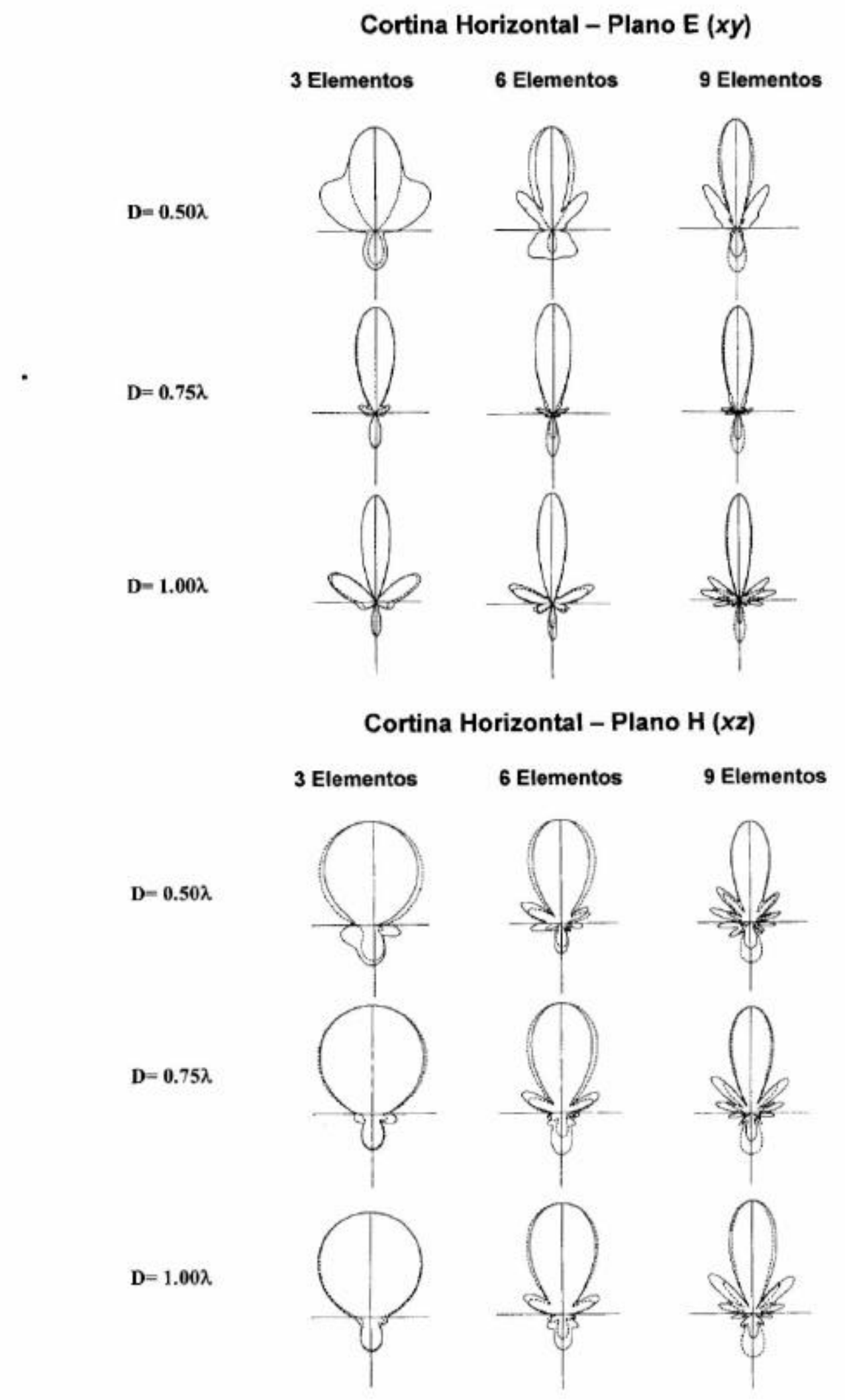

Figura 7. Patrón de radiación de cortinas horizontales con Yagis de 3, 6 y 9 elementos (---Multiplicación de patrones, Método de momentos]. Todas las intensidades de campo eléctrico de cada patrón están normalizadas con relación a su 

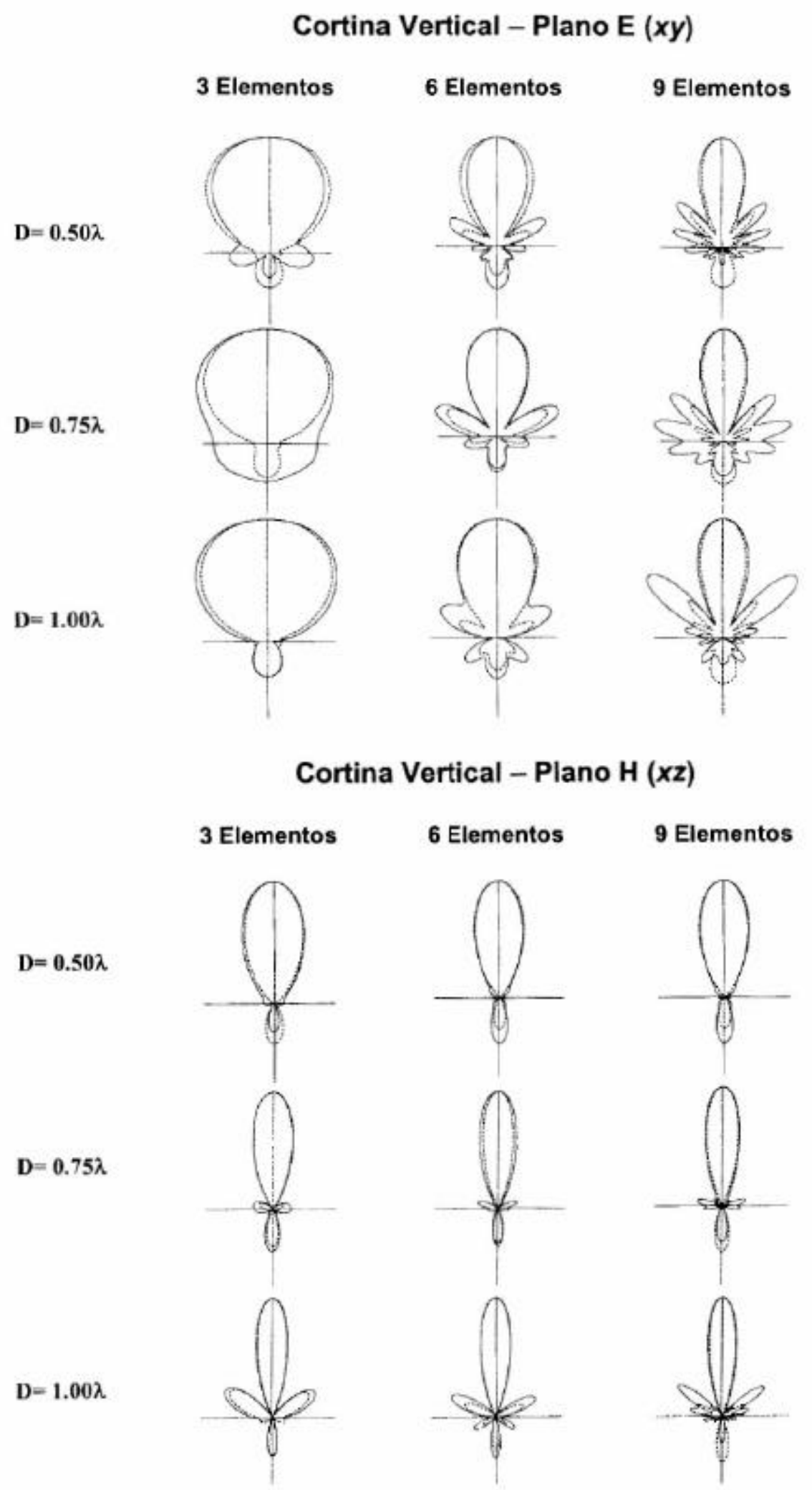

Figura 8. Patrón de radiación de cortinas verticales con Yagis de 3, 6 y 9 elementos (--- Multiplicación de patrones, Método de momentos). Todas las intensidades de campo eléttrico de cada patrón están normalizadas con relación a su campo máximo 


\section{Método de multiplicación de patrones}

El método de multiplicación de patrones permite encontrar el patrón de radiación aproximado de un arreglo, pero su utilidad se reduce solamente a eso y no es posible obtener mediante él las demás características operativas de la antena, tales como su ganancia e impedancia de entrada. A continuación se describe brevemente cómo calcular dicho patrón aproximado por el método de multiplicación de patrones.

El campo lejano de radiación de un arreglo de elementos idénticos (antenas elementales) es igual al producto del campo producido por uno solo de estos elementos, localizado en un punto de referencia determinado (usualmente el origen del sistema de referencia), y el factor del arreglo. Es decir:

$\mathrm{E}($ total $)=[\mathrm{E}($ elemento único en el punto de referencia)]x [factor del arreglo]

A esta relación se le conoce como. multiplicación de patrones para arreglos de elementos idénticos (Balanis, 1997), ya que al evaluar la ecuación (3) en muchos puntos alrededor del arreglo, es posible obtener su patrón de radiación. En la figura 9 se muestran arreglos de tres elementos isotrópicos separados una distancia D y con magnitudes de alimentación $a_{n}$ no uniformes. A partir de dichas geometrías se puede obtener el factor del arreglo (FA) tanto para la cortina horizontal (Figura 9a) como para la vertical (Figura 9b). En ambos casos se utilizaron las magnitudes normalizadas $a_{B}=1 \mathrm{y}$ $\mathrm{a}_{\mathrm{A}}=\mathrm{a}_{\mathrm{c}}=\mathrm{a}_{\mathrm{B}} / 2=0.5$, de acuerdo con el circuito de alimentación de la figura 4 y suponiendo un mismo voltaje de excitación en las antenas A y $\mathrm{C}$, aun cuando en la cortina vertical, su impedancia de entrada varía en algunos Ohms por la asimetría de la alimentación en el arreglo (Figura 3), tal como se comentó anteriormente. Para un punto en el campo lejano en una dirección $\Phi$, el factor del arreglo en el plano $x y$ de la figura $9 \mathrm{a}$ (cortina horizontal) está dado por:

$$
(\mathrm{FA})_{\text {Cortina Harizonces }}=\frac{a_{\mathrm{B}}}{2}+a_{\mathrm{A}} \cos \left(\frac{2 \pi \mathrm{D}}{\lambda} \cos \phi\right)
$$

y para encontrar el factor del arreglo en el plano $x z$ de la figura 9b (cortina vertical) es suficiente intercambiar el ángulo $\Phi$ en la ecuación 4 por el ángulo $\Theta$, como se muestra en la siguiente ecuación:

$$
(\mathrm{FA})_{\text {Corrisavertian }}=\frac{a_{\mathrm{B}}}{2}+a_{\mathrm{A}} \cos \left(\frac{2 \pi \mathrm{D}}{\lambda} \cos \theta\right)
$$

En las figuras 7 y 8 se muestran los patrones de radiación normalizados para cortinas horizontales y verticales respectivamente, con 3,6 y 9 elementos y con separaciones $D$ entre antenas Yagi de $0.5 \lambda, 0.7 \lambda$, y $1.0 \lambda$ obtenidos mediante el método de momentos (-) y también por el método de multiplicación de patrones ( - - ) .

Se nota que, en todos los casos, la diferencia entre los patrones predichos por cada método es mayor conforme $D$ es más pequeña; esto se debe a que el efecto de las impedancias mutuas es mayor para estas separaciones pequeñas, por la cercanía de los conductores vecinos, y esto no lo toma en cuenta el método de multiplicación de patrones, mientras que el método de momentos sí incluye estas impedancias mutuas. Sin embargo, hay que resaltar que el método de multiplicación de patrones predice, en todos los casos, el mismo número de lóbulos laterales y en las mismas direcciones que el método de momentos, pero con algunas diferencias importantes de magnitud, tanto en estos lóbulos como en el posterior. De allí que, al efectuar dicha comparación, es posible determinar un límite para la separación $D$ donde sea permisible usar el método de multiplicación de patrones, el cual aporta resultados muy rápidos mediante cálculos muy sencillos. 


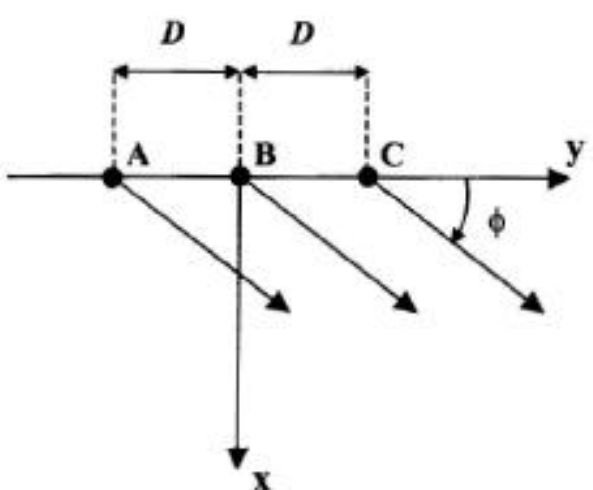

a)

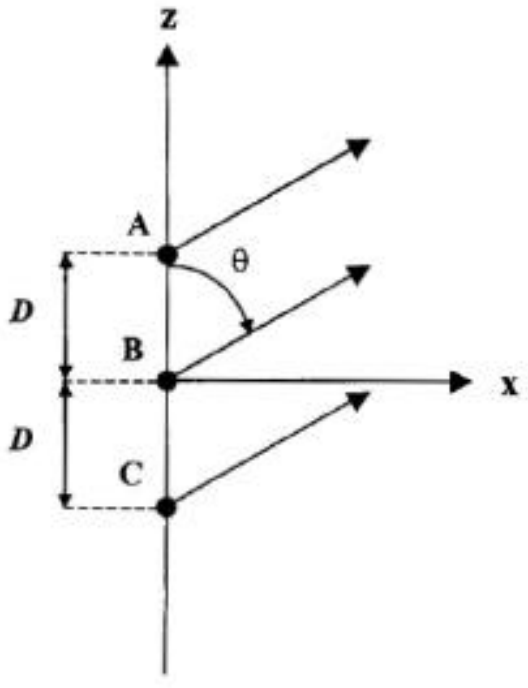

b)

Figura 9. Arreglo de tres fuentes isotrópicas: a) sobre el eje y para modelar una cortina horizontal (véase la figura 2): b) sobre eje z para modelar una cortina vertical (véase la figura 3)

\section{Conclusiones}

El método aproximado de multiplicación de patrones permite obtener el patrón de radiación de arreglos de tres antenas Yagi (troicas) con cierta precisión, siempre y cuando la distancia entre ellas no sea muy pequeña. Para usar este método se recomienda que la separación D sea igual o mayor que $0.75 \lambda$. Sin embargo, resulta impráctico desde el punto de vista de montaje, altura de mástiles o extensión de terreno, construir arreglos con separaciones mayores, y por ello, es necesario usar un método más preciso para conocer las características de operación de arreglos prácticos que usan separaciones menores.

Esto se logra usando el método de momentos, mismo que al tomar en cuenta todos los acoplamientos mutuos de impedancia entre los conductores de las Yagis del arreglo, da resultados mucho más aproximados a la realidad. Este método tiene, además, la virtud de que no sólo permite calcular el patrón de radiación sino también la impedancia de entrada, que es información necesaria para alimentar la antena con óptimos resultados. En este trabajo se ha demostrado que la ganancia de los arreglos estudiados es mayor que la de una antena Yagi aislada, entre 2 y $4 \mathrm{dBs}$ más, según el número de conductores que tenga cada Yagi.
Por ejemplo, la ganancia de una troica con Yagis de un solo director es equivalente a la ganancia de una sola antena Yagi con siete directores. Sin embargo, el montaje de esta última es más complicado y puede ser un factor en contra de su uso. También se ha encontrado que las curvas de ganancia de las troicas horizontales y verticales son muy parecidas, pero que existe una diferencia importante en el funcionamiento de dichos tipos de troica en lo que se refiere al comportamiento de su VSWR, en función de la frecuencia. Esto es importante para determinar la alimentación óptima del arreglo porque el VSWR está directamente asociado con su impedancia de entrada, ya que al depender de la frecuencia influye sobre el ancho de banda de operación. En este sentido, el ancho de banda de una cortina horizontal es mayor que el de una vertical, aproximadamente un $40 \%$.

El elegir un tipo de troica sobre otro, depende del patrón de radiación con el que se desee trabajar,ya que una cortina horizontal tiene un ancho de haz en su lóbulo principal que es menor en el plano horizontal que el de una Yagi sola, mientras que una cortina vertical tiene un ancho de haz de su lóbulo principal que es menor en el plano vertical que el de una antena Yagi sola. 
DOI: http://dx.doi.org/10.22201/fi.25940732e.2005.06n2.006

R. Neri-Vela, L.A. Valiente-Montaño y V. Hernández-Solís

\section{Referencias}

Balanis C.A. (1997). Antenna Theory, Analysis and Design. Second Edition, John Wiley \& Sons, Inc., EUA.

Collin R.E. (1985). Antennas and Radiowave Propagation. McGraw Hill International Editions. EUA.

Decibel Products (2000). Base Station Antennas and RF Components. Product Catalog. Decibel Products, Dallas, Texas, EUA.

Harrington R.F. (1968). Field Computation by

Moment Methods. Macmillan, Nueva York.
Hernández V., Valiente L.A. Y Neri R. (2002). The Method of Moments. Http://telecom.fib.unam.mx/weblink/TheMethod of Moments.htm

Kraus J.D. y Marhefka R.J. (2002). Antennas for all Applications. Third Edition. McGraw Hill, EUA. Neri R. (1999). Análisis de Antenas Lineales por el Método de Momentos. Ingeniería Investigación y Tecnología, UNAM. Vol. 1, No. 2, pp.73-83. Rossier H. (1986). Antenas Colectivas. Boixareu Editores, Barcelona, México.

\section{Semblanza de los autores}

Rodolfo Neri-Vela. En 1975, obtuvo su título de ingeniero mecánico-electricista en el área de comunicaciones y electrónica por la Facultad de Ingeniería de la UNAM. En 1976, recibió el grado de maestría en sistemas de telecomunicaciones otorgado por la Universidad de Essex, Reino Unido. En 1979, alcanzó el grado de doctorado en electromagnetismo aplicado por parte de la Universidad de Birmingham, también en el Reino Unido. En 1985, se convirtió en el primer astronauta de México, al participar en la misión 61-B de la NASA de los EU y orbitar la Tierra 109 veces. Es profesor e investigador del Departamento de Telecomunicaciones de la Facultad de Ingeniería de la UNAM desde hace 23 años y pertenece al Sistema Nacional de Investigadores del CONACYT.

Luis Alán Valiente-Montaño. Obtuvo su título en el año 2000 como ingeniero en telecomunicaciones por la Facultad de Ingeniería de la

UNAM . En el 2003, recibió el grado de maestría en ingeniería de comunicaciones otorgado por el Instituto de Ciencia y Tecnología de la Universidad de Manchester (UMIST), Reino Unido. Actualmente está becado por el CONACYT y realiza sus estudios de doctorado en la misma Universidad de Manchester, en el área de microscopía por microondas en el campo cercano.

Vladimir Hernández-Solis. Se tituló como ingeniero en telecomunicaciones en el 2000, en la Facultad de Ingeniería de la UNAM. En el año 2003 recibió el grado de maestría en ingeniería de comunicaciones, otorgado por el Instituto de Ciencia y Tecnología de la Universidad de Manchester (UMIST), Reino Unido. Tiene experiencia en arreglos adaptivos para redes celulares CDMA, antenas y óptica no lineal. Actualmente realiza sus estudios de doctorado en el Centro de Investigaciones en Optoelectrónica de la Universidad de Southampton, Reino Unido, en el área de amplificadores de fibra de alta potencia. 